\title{
Optimal stimulation parameters for intraoperative bulbocavernosus reflex in infants
}

\author{
Hyeoncheol Hwang, MD, ${ }^{1,2}$ Kyu-Chang Wang, MD, PhD, ${ }^{3}$ Moon Suk Bang, MD, PhD, ${ }^{1}$ \\ Hyung-lk Shin, MD, PhD, ${ }^{1}$ Seung-Ki Kim, MD, PhD, ${ }^{3}$ Ji Hoon Phi, MD, PhD, ${ }^{3}$ \\ Ji Yeoun Lee, MD, PhD, ${ }^{4}$ Jinwoo Choi, BS, ${ }^{1}$ Seungwoo Cha, MD, ${ }^{1}$ and Keewon Kim, MD, PhD ${ }^{1,2}$ \\ Departments of ${ }^{1}$ Rehabilitation Medicine, ${ }^{2}$ Biomedical Engineering, ${ }^{3}$ Neurosurgery, and ${ }^{4}$ Anatomy, Seoul National University \\ College of Medicine, Seoul National University Hospital, Seoul, Republic of Korea
}

\begin{abstract}
OBJECTIVE The aim of this study was to establish optimal electric stimulation parameters for intraoperatively monitoring the bulbocavernosus reflexes (BCRs) in infants.

METHODS The authors retrospectively reviewed the medical records of all infants (age $<24$ months) who had undergone an untethering operation for tethered cord syndrome between May 2013 and February 2014 at a single institution and whose baseline BCR had been elicited during surgery. Using different combinations of stimulation parametersnumber of stimulation pulses: 4 or 8 pulses, interpulse interval: 1, 2, or $5 \mathrm{msec}$, and polarity of stimulation: biphasic or monophasic - the authors compared the relative mean amplitude of $10 \mathrm{BCR}$ responses (rmaBCRs) to each combination of parameters.
\end{abstract}

RESULTS The rmaBCRs were larger with the 8-pulse stimulations than with the 4-pulse stimulations $(p<0.0001)$. There was a tendency, though not statistically significant, for larger rmaBCRs to be obtained with the longer interpulse interval in the 8-pulse stimulation $(p=0.1289)$. The biphasic stimulation produced larger rmaBCRs than the monophasic stimulation $(p=0.0005)$.

CONCLUSIONS Biphasic 8-pulse stimulations with 5-msec or 2-msec intervals yield the largest BCR responses. Considering that an 8-pulse stimulation with 5-msec intervals may overlap the onset of the BCR, a biphasic 8-pulse stimulation with 2-msec intervals is recommended as the optimal stimulation paradigm to monitor intraoperative BCRs in infants. https://thejns.org/doi/abs/10.3171/2017.6.PEDS16664

KEY WORDS intraoperative monitoring; bulbocavernosus reflex; infants; tethered cord syndrome; spinal dysraphism; electric stimulation; surgical technique; spine

$\mathrm{P}$ RESERVING sacral function during surgery for tethered cord syndrome and spinal dysraphism is of great clinical importance. ${ }^{5}$ Further, monitoring the bulbocavernosus reflex (BCR) is useful because it can reflect the integrity of the complex sensorimotor reflex arc within the sacral root level and sphincter control. ${ }^{6,8}$ Moreover, the BCR can be elicited and recorded outside the surgical field, without gross movement, thereby enabling continuous monitoring during surgery. ${ }^{2}$

Most cases of congenital tethered cord syndrome, with or without spinal dysraphism, are diagnosed in infants or children, and early surgery is favored because neuro- logical deficits are usually progressive and irreversible. ${ }^{4,12}$ Also, a young age correlates with a better progression-free survival probability. ${ }^{11}$ Therefore, an intraoperative BCR has clinically significant value in young children.

However, many surgeons and neurophysiologists experience difficulties in obtaining a BCR intraoperatively in infants under the age of 24 months. The conventional stimulation paradigm for the largest BCR response is monophasic (cathode proximal) stimulation, consisting of 4 or 5 pulses with 3-msec intervals, of the penis or clitoris., ${ }^{2,13}$ That paradigm has been developed mainly for adults and is known to be applicable in children older than 24 months. ${ }^{1}$

ABBREVIATIONS BCR = bulbocavernosus reflex; GEE = generalized estimating equation; rmaBCR = relative mean amplitude of the $B C R$. SUBMITTED November 30, 2016. ACCEPTED June 6, 2017.

INCLUDE WHEN CITING Published online September 1, 2017; DOI: 10.3171/2017.6.PEDS16664. 
However, the usual stimulation paradigm frequently fails to generate an effective BCR in infants younger than 24 months.

The objective of the present study was to investigate the optimal stimulation parameters for obtaining a BCR in infants under the age of 24 months during an untethering operation. The BCR amplitudes were compared for different pulse numbers, interpulse intervals, and pulse polarities (biphasic vs monophasic).

\section{Methods}

\section{Subjects}

The medical records of patients who had undergone an untethering operation for tethered cord syndrome between May 2013 and February 2014 at Seoul National University Children's Hospital were reviewed retrospectively. Patients were included in the study if 1) they had an age less than 24 months and 2) they had undergone untethering surgery. Patients were excluded if their baseline BCR could not be obtained; the baseline BCR was not elicited in a proportion of patients with complex or severe lesions who exhibited bladder or bowel dysfunction. Ultimately, 12 patients (6 males) were included in our analysis (Table 1). Patients in the study cohort were 3-23 months old (median age 6.5 months) and had been diagnosed with lumbosacral lipomatous malformation (8 cases), limited dorsal myeloschisis (2), congenital dermal sinus (2), or Currarino's triad (1). In this study, lumbosacral lipomatous malformation was further classified as follows: thick filum terminale (filum terminale remains intact but is wrapped by a lipoma, 2 cases), lumbosacral lipoma (lipoma that is integrated with the neural tissue, which has not extended to the extraspinal space, 5 cases), or lipomeningomyelocele (dorsal extraspinal extension of a complex lipoma mixed with neural tissue, 1 case). The study was approved by the institutional review board of Seoul National University Hospital.

\section{Anesthesia}

Total intravenous anesthesia was induced using propo- fol and remifentanil in all patients. Muscle relaxants were used only for intubation, and train-of-four monitoring was used for confirming that the residual effects of these relaxants did not remain.

\section{The BCR Technique}

Surface electrodes were used for the stimulation (cathode on the proximal penis or clitoris; anode on the distal penis or labia majora), which consisted of $75-\mu$ sec squarewave pulses. The intensity of stimulations was determined for each patient at the submaximal level and was kept constant for the patient. For the recording, subdermal needle electrodes were placed at the anal sphincter except in 1 patient with Currarino's triad (Case 2) in whom the needle electrode was placed at the bulbocavernosus because of a deformity of the anus (Fig. 1). The NIM-Eclipse NS system (Medtronic Xomed Inc.) was used for intraoperative monitoring.

\section{Independent Variables (Stimulation Parameters) and Outcomes}

For the clinical purpose of monitoring the BCR response in proper stimulation conditions, 9 combinations of 3 kinds of stimulation parameters had been tested for each patient. The 3 varying stimulation parameters were the number of stimulation pulses (4 or 8 pulses), the interpulse interval $(1,2$, or $5 \mathrm{msec}$ ), and the pulse polarity (biphasic or monophasic current; Fig. 2). All 6 possible combinations with biphasic stimulation and 3 combinations with monophasic stimulation (4 pulses of 2-msec intervals, 8 pulses of 2-msec intervals, and 8 pulses of 1-msec intervals) were tested. Ten BCR responses were obtained for each stimulation condition.

The efficacy of each combination of stimulation parameters for a subject was determined using the mean amplitude of $10 \mathrm{BCR}$ responses to the parameter combination. To allow comparison among the subjects, the mean amplitude of the BCR was converted into the relative mean amplitude of the BCR (rmaBCR). An rmaBCR value of a

TABLE 1. Demographic and clinical information on 12 patients with tethered cord syndrome

\begin{tabular}{ccclll}
\hline Case No. & Age $(\mathrm{mos})$ & Sex & \multicolumn{1}{c}{ Diagnosis } & Operation & Postop Voiding Function \\
\hline 1 & 16 & F & Congenital dermal sinus & Untethering w/ mass excision & Normal \\
\hline 2 & 23 & F & Currarino's triad & Untethering w/ mass excision & Normal \\
\hline 3 & 4 & F & Limited dorsal myeloschisis & Untethering & Normal \\
\hline 4 & 8 & M & Thick filum terminale & Untethering & Normal \\
\hline 6 & 10 & F & LS lipoma & Untethering w/ mass excision & Normal \\
\hline 7 & 10 & M & LS lipoma & Untethering w/ mass excision & Timed CIC (recovered to normal \\
& & & & Untethering w/ mass excision & Normal \\
\hline 8 & 5 & M & Thick filum terminale & Untethering w/ mass excision & Normal \\
\hline 10 & 4 & F & Lipomeningomyelocele & Untethering & Normal \\
\hline 11 & 3 & M & Congenital dermal sinus & Untethering w/ mass excision & Normal \\
\hline 12 & 4 & M & LS lipoma & Untethering & Normal \\
\hline
\end{tabular}

$\mathrm{CIC}=$ clean intermittent catheterization; $\mathrm{LS}$ lipoma = lumbosacral lipoma. 


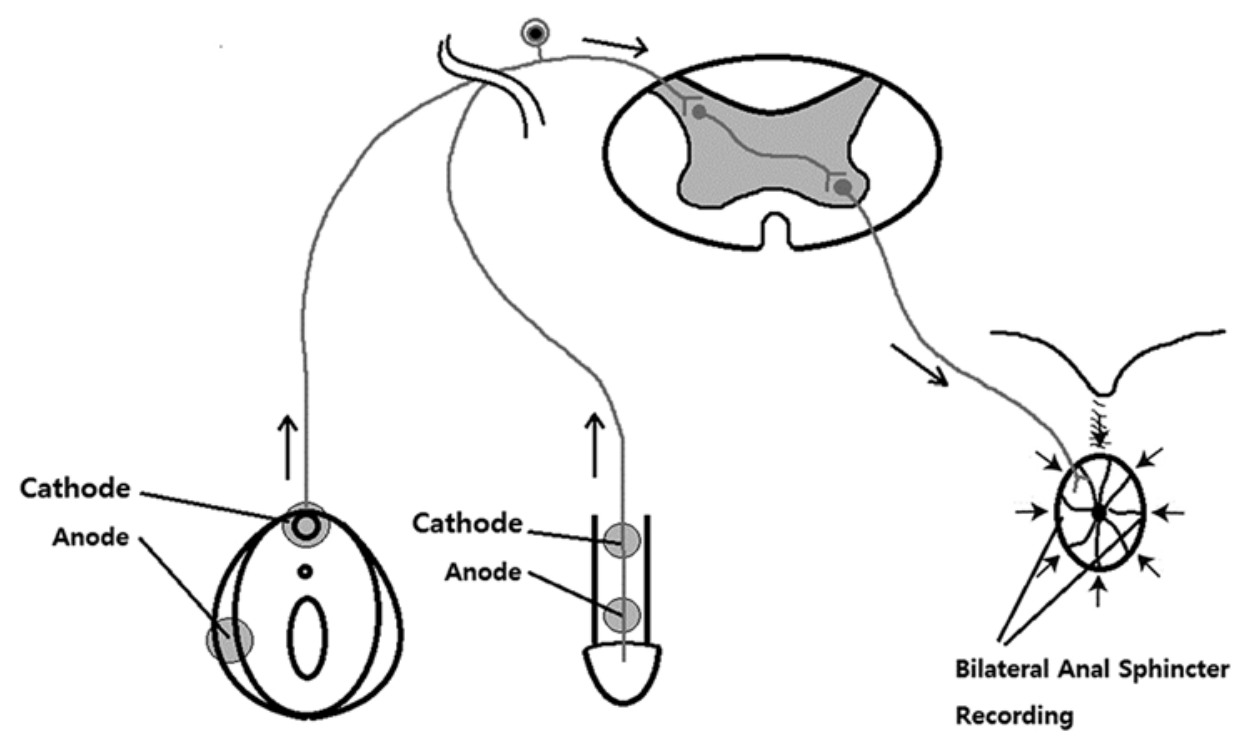

FIG. 1. Schematic representation of the stimulation recording: sacral segment of spinal cord (upper), male and female anatomy (lower left), and anal sphincter (lower right).

stimulation condition was obtained by calculating the ratio of the mean BCR amplitude with the stimulation condition to the subject's maximal BCR amplitude throughout all the stimulation conditions.

\section{Statistical Analysis}

The effect of different numbers of pulses and interpulse intervals on the rmaBCR in the biphasic stimulation was evaluated using the generalized estimating equation (GEE) method. The GEE method enables estimation of the population-averaged response in repeated tests. The varying trend of the rmaBCR according to the interpulse interval was also assessed using GEE methods. Biphasic and monophasic stimulations were also compared for 3 combinations of the other parameters (2-msec interval, 4 pulses; 2 -msec interval, 8 pulses; 1-msec interval, 8 pulses) in terms of the rmaBCR by using GEEs. Statistical analysis was conducted with SAS version 9.2 (SAS Institute Inc.).

\section{Results}

\section{Optimal Stimulation Paradigm}

The 8-pulse stimulation elicited a larger rmaBCR than the 4-pulse stimulation $(0.6098 \pm 0.0402$ and $0.4368 \pm$ 0.0365 , respectively; $\mathrm{p}<0.0001$ ) with biphasic current stimulations at 3 different interpulse intervals. When separately evaluated for each interpulse interval, the rmaBCR difference between the 8-pulse and the 4-pulse stimulations was statistically significant in the case of the 5-msec interpulse interval $(\mathrm{rmaBCR}$ difference $=0.24, \mathrm{p}=0.0075)$ but not in the case of the 1-msec interval (rmaBCR difference $=0.11, p=0.0660)$ or 2-msec interval (rmaBCR difference $=0.16, p=0.0604 ;$ Fig. 3).

There was a trend toward eliciting a larger rmaBCR with a longer interpulse interval in the 8-pulse stimulations (rmaBCR $=0.5423,0.6209$, and 0.6662 for interpulse intervals of 1, 2, and $5 \mathrm{msec}$, respectively; Fig. 3); how- ever, it was not statistically significant $(p=0.1289)$. In the 4-pulse stimulations, there was no significant difference in the rmaBCR between the different interpulse intervals.

Larger BCR amplitudes were obtained with biphasic stimulations than with monophasic stimulations under all 3 examined conditions ( $\mathrm{rmaBCR}=0.55$ and 0.46 , respectively, $\mathrm{p}=0.0005$; Fig. 4).

\section{Illustrative Cases}

\section{Case 10}

A boy, born by normal full-term spontaneous delivery, had a skin dimple with proboscis at the sacral area. Magnetic resonance imaging revealed a lumbosacral lipoma with syrinx and tract (Fig. 5A). Preoperative evaluation revealed no definite abnormality in motricity of the lower

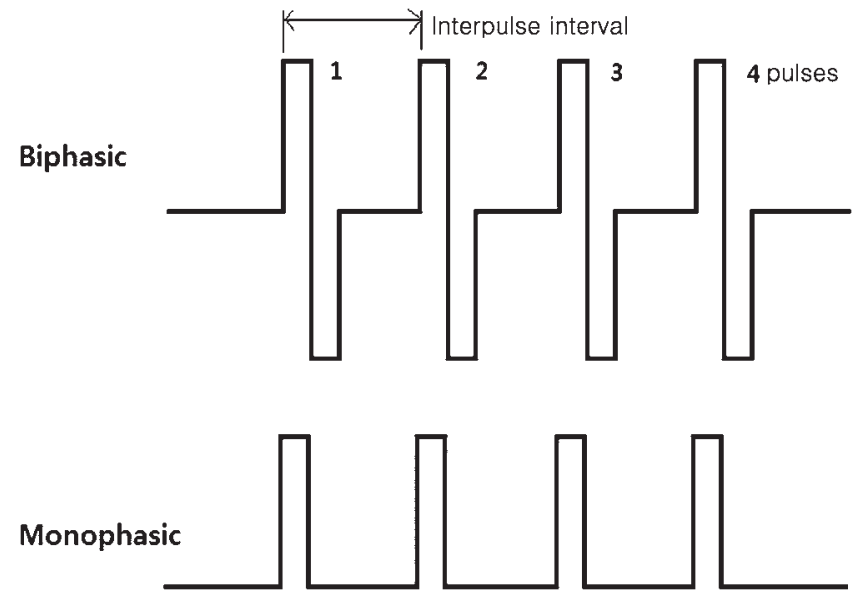

FIG. 2. The stimulation pulse waveform. Stimulation consisted of 4 or 8 (not shown in figure) square-wave pulses. The duration of a squarewave pulse was $75 \mu \mathrm{sec}$, and the interpulse interval was 1, 2, or 5 msec. The amplitude of stimulation was individually determined at the submaximal level. 


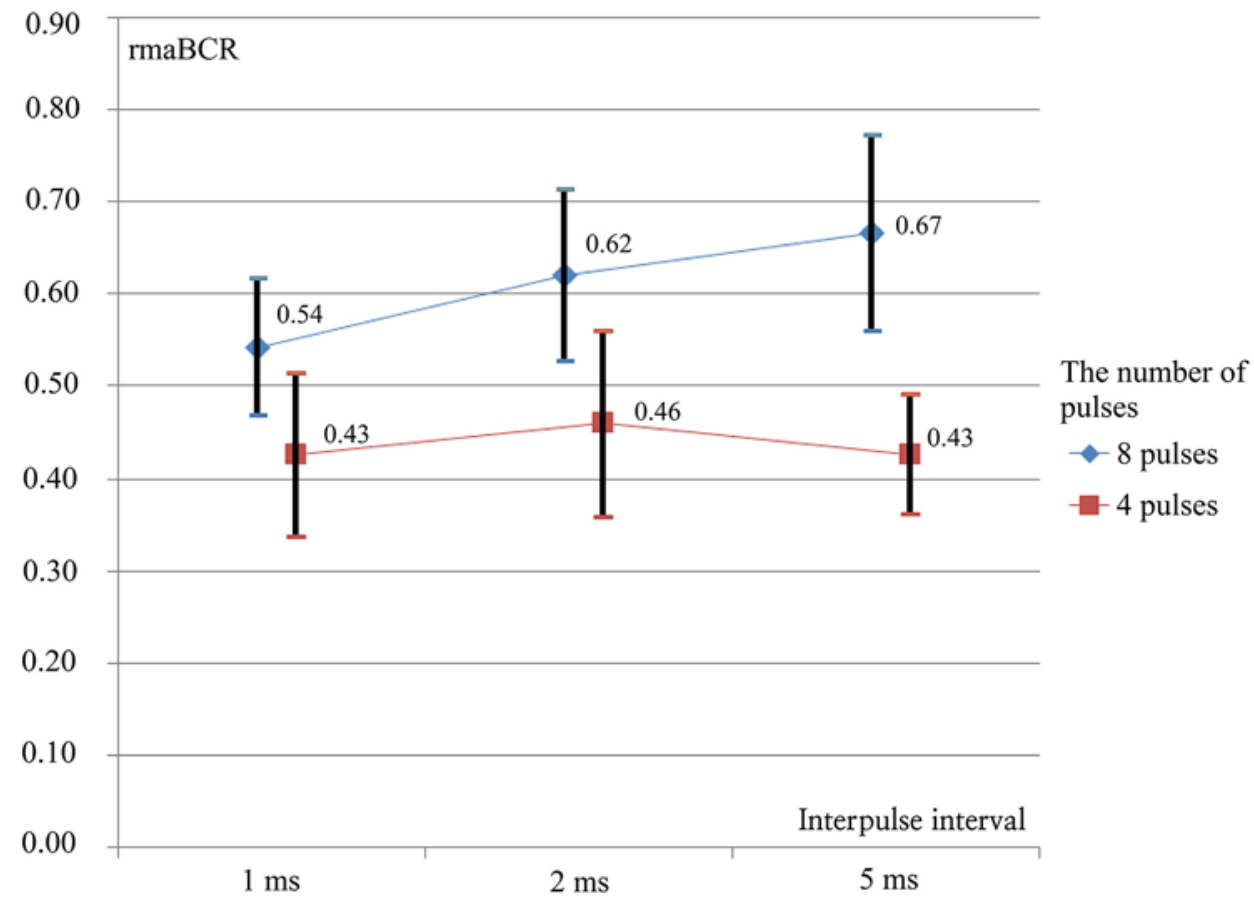

FIG. 3. The effect of pulse number and interpulse interval on the relative mean amplitude of the BCR (rmaBCR). Biphasic 8-pulse stimulation produced a larger rmaBCR than biphasic 4-pulse stimulation with a statistically significant difference $(p<0.0001$, GEE under all interpulse intervals), particularly under 5-msec interpulse interval conditions (adjusted $p=0.0075$, Hochberg post hoc analysis). The stimulation with a longer interpulse interval tended to elicit a larger rmaBCR only in the 8-pulse condition, but the trend was not statistically significant $(p=0.1289)$. Figure is available in color online only.

extremities, the electromyography study (including external anal sphincter), or the urodynamic study. The boy underwent untethering surgery (complete removal of the lipoma) at the age of 4 months. During surgery, the BCR was efficiently obtained with a biphasic 8-pulse stimulation with 2-msec interpulse intervals and was monitored

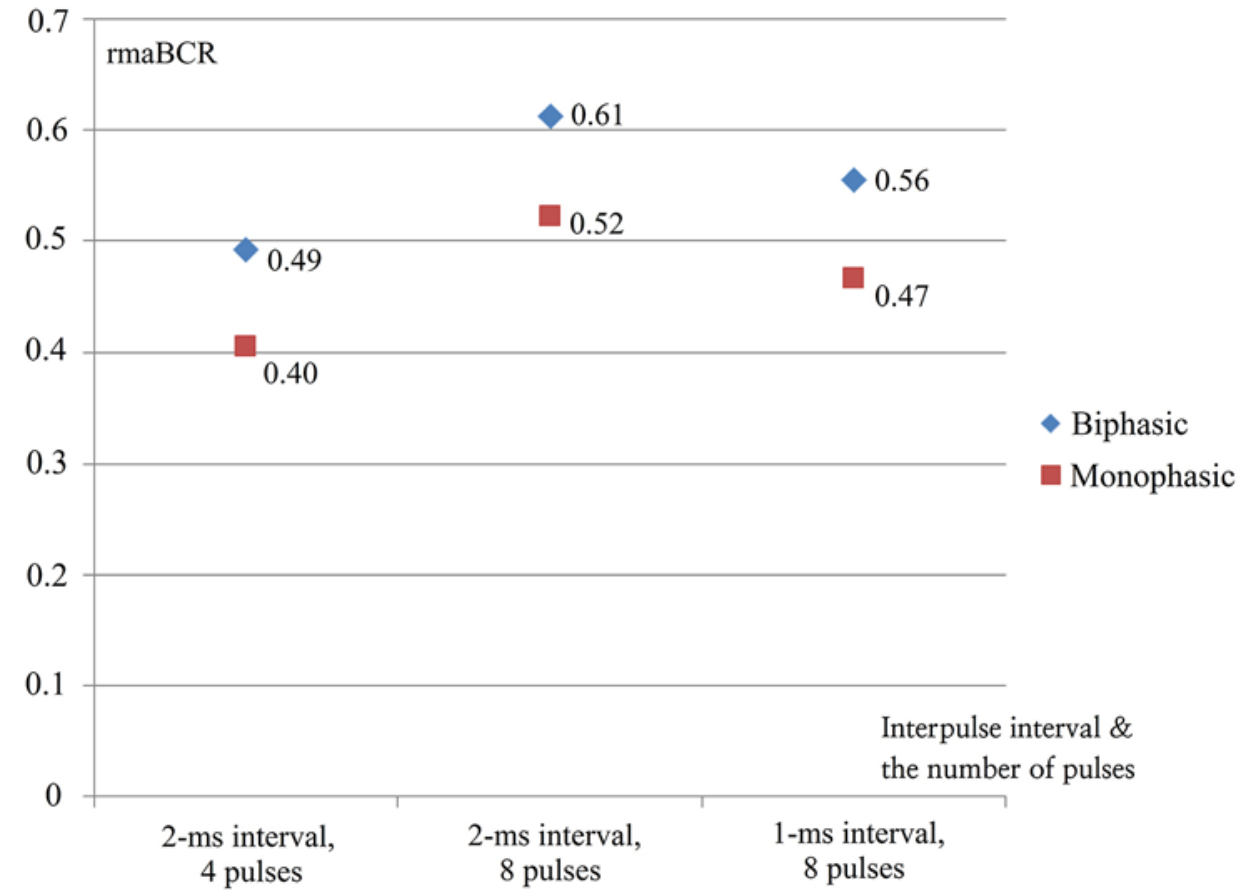

FIG. 4. The effect of pulse polarity (biphasic or monophasic) on the rmaBCR. Biphasic stimulations yielded a significantly larger rmaBCR than monophasic stimulations regardless of the number of pulses or the interpulse interval $(p=0.0005)$. Figure is available in color online only. 


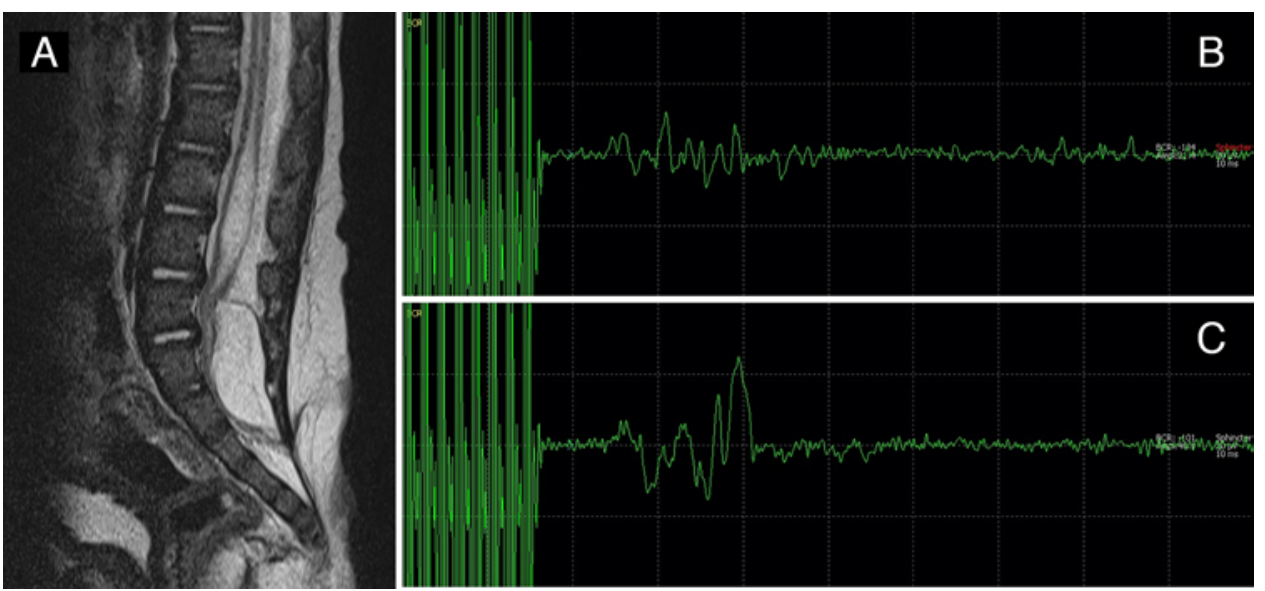

FIG. 5. Case 10. A male infant was diagnosed with lumbosacral lipoma and underwent untethering surgery at the age of 4 months. A: An MR image revealed the lumbosacral lipoma with syrinx and tract. B: The baseline BCR obtained from a biphasic 8-pulse stimulation with 2-msec interpulse intervals. C: The BCR at the end of the surgery. Postoperatively, voluntary voiding function was maintained, and postvoiding residue was negligible. Figure is available in color online only.

while maintaining that stimulation paradigm. The BCR was maintained without attenuation from baseline (Fig. 5B) until the end of surgery (Fig. 5C). Postoperatively, voluntary voiding was preserved without significant postvoiding residue.

\section{Case 13}

A girl, born via normal vaginal delivery at 39 gestational weeks, had a sacrococcygeal dimple with skin defect and was diagnosed with limited dorsal myeloschisis (Fig. 6A). She demonstrated no neurological deficit. Preoperative electromyography and urodynamic studies were normal. She underwent untethering at the age of 3 months. Baseline BCR was apparent (Fig. 6B) with a biphasic 8-pulse stimulation with 2-msec interpulse intervals; however, the BCR disappeared during untethering and did not recover until the end of surgery (Fig. 6C). Lower-extremity motor evoked potentials and the tibial sensory evoked potential were maintained. The girl was dependent on clean intermittent catheterization for 1 month following the operation. Thereafter, voluntary voiding was gradually regained, although postvoiding residue was significant and fecal continence was not acquired until recently (4 years old). Her motor function for walking and running was normal. (Note that this case was not included in the study and its description appears merely for illustrative purposes.)

\section{Discussion}

In the present study, the largest BCR amplitude was obtained in the biphasic 8-pulse stimulation with 5-msec interpulse intervals. In the literature, 4-pulse or 5-pulse stimulation with 3-msec intervals has been reported to be the optimal stimulation condition for a BCR., ${ }^{213}$ However, the stimulation paradigms in these previous studies were based on adult data and have not been found to be applicable to infants. In the present study, we showed that the stimulation condition required for infants younger than 24 months involves more pulses and a longer interpulse interval than those in adults. In fact, our clinical experi-
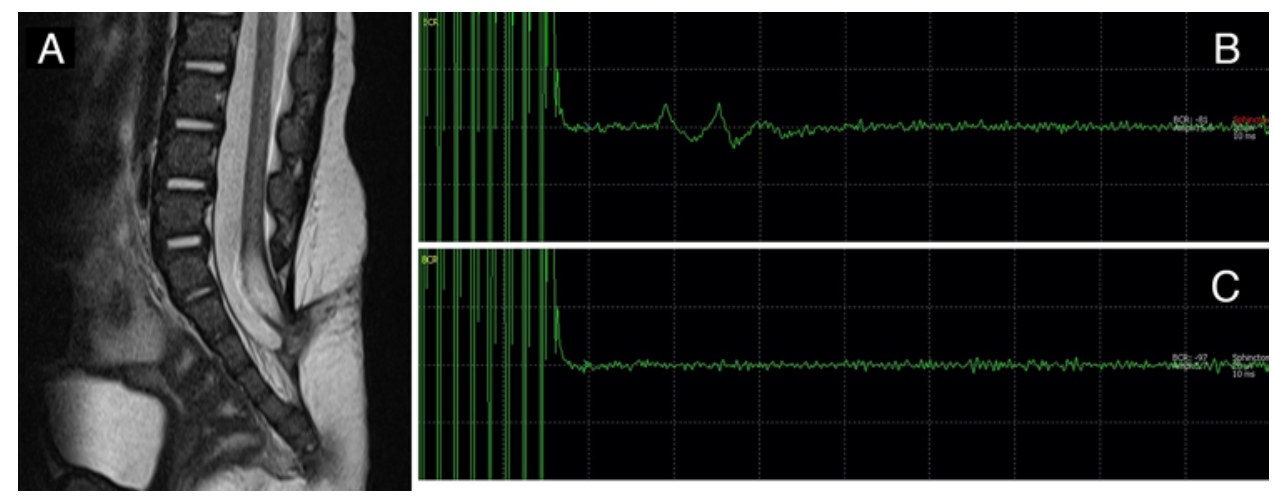

FIG. 6. Case 13. A female 3 months of age was diagnosed with limited dorsal myeloschisis associated with lipoma and underwent untethering surgery. A: An MR image revealed limited dorsal myeloschisis. B: The baseline BCR elicited from a biphasic 8-pulse stimulation with 2-msec interpulse intervals. C: The BCR at the end of surgery disappeared. Postoperatively, clean intermittent catheterization was required for a month. Fecal incontinence remained until recently (4 years old). Figure is available in color online only. 
ence was in accordance with the literature on cases of older children: stimulation with 2-msec interpulse intervals generated a larger BCR amplitude than stimulation with 5-msec interpulse intervals in children older than 24 months (data not shown).

Differences in the optimal stimulation conditions are thought to be caused by the neurophysiological differences between infants and adults. Myelination of nerves is not complete in infants; therefore, neural excitabilities are lower and nerve conduction velocities are slower than those in adults. Nerves in infants are relatively small in size and have a more unstable physiology. These differences may call for stimulation with more pulses and longer intervals.

However, the 8-pulse stimulation with 5-msec intervals may not always be applicable because of its long stimulation duration. The stimulation artifact can overlap the onset of the BCR because the BCR onset latency is about $30-40 \mathrm{msec}(36.3 \pm 10.44 \mathrm{msec}$ in the present study). Therefore, the 8-pulse stimulation with 2-msec intervals was more practically feasible than the 8-pulse stimulation with 5-msec intervals with respect to observing entire waveforms of the BCR. In practice, it is better to individualize the pulse interval between 2 to $5 \mathrm{msec}$ to obtain the largest BCR response as well to avoid overlap of the BCR with the stimulation artifact.

A monophasic pulse delivers only a negative pulse, while a biphasic pulse delivers a negative pulse immediately followed by a positive pulse (Fig. 2). In general, monophasic stimulation is considered the standard method for monitoring the BCR, either during intraoperative monitoring or in the electromyography room. ${ }^{7}$ However, a biphasic pulse is safer for repeated stimulation since it poses less risk of charge accumulation. ${ }^{14}$ Meanwhile, the effective pulse duration of a biphasic stimulation is half that of a monophasic stimulation. Thus, the effective stimulation intensity of a biphasic stimulation is lower than that of a monophasic stimulation, although it is at the same voltage or current. ${ }^{3}$ In the current study, biphasic stimulation generated larger BCR responses than did monophasic stimulation. We presumed that the conventional method in the electromyography room was replicated in the intraoperative monitoring, without further investigation. Nonetheless, the efficacy and safety of biphasic stimulation for the electromyography room requires further study in other age groups or in awake patients.

Even with optimal stimulation, the intraoperative BCR is not always obtainable. For instance, a baseline BCR may not be obtainable in patients whose lesions are complicated by sacral dysfunction or in those who are too young (younger than 1-2 months old) since their peripheral nervous system is not mature enough to be monitored. Moreover, technical inadequacy (for example, insufficient stimulation, misplaced recording or stimulation electrodes, stimulation artifacts) may preclude acquisition of a BCR response in young children, which was the motivation for the current study. Given the development of optimal stimulation parameters for young children, in the absence of obtaining a BCR, we often suspect preexisting sacral radiculopathies but rarely suspect technical inadequacy. If the baseline BCR is not obtainable, sacral function can be monitored using the sensory evoked potential (SEP) of the pudendal nerve and the motor evoked potential (MEP) of the external anal sphincter. However, since they involve longer nervous system tracts, they are more prone to systemic perturbation. Further, the SEP takes approximately 30 seconds to elicit a response and the MEP can produce movement artifact, thereby limiting their application for continuous monitoring.

If the BCR or other neurophysiological signals are lost or reduced during untethering, the deterioration may be reversible or irreversible. To correct a reversible deterioration, surgeons should cease the current procedure and inspect the surgical field for possible insult on the neural tissue such as compression, traction, or clamping. If any surgical procedure is suspected to be causing injury to or compromise of the nervous system and thus a loss of the $\mathrm{BCR}$, it should be stopped. If the BCR recovers thereafter, the operation can be resumed with caution. However, if surgeons or neurophysiologists cannot identify the specific or local cause, they should review possible systemic causes, such as the depth of anesthesia, infusion of a muscle relaxant, or low blood pressure. In addition to the correction of those systemic factors, warm saline irrigation can also be attempted. ${ }^{9}$ In most cases, intraoperative signals are recovered to the baseline without permanent injury of the nervous system. When signals are not recovered, surgeons should be aware of the neurological compromise and decide whether to advance the operation, balancing the risk of possible neurological deficit with the benefit of further debulking. The irreversible disappearance of the BCR suggests the development of postoperative sacral dysfunction. In our experience, maintenance of the BCR is highly predictive of normal bladder function. A proportion of patients whose BCR was lost developed a postoperative voiding problem (data not published). This may indicate that the BCR is a sensitive measure of the sacral nervous system. The sensitivity and specificity of the BCR are currently under investigation.

The present study has several limitations. First, its sample size was small. In particular, the trend of an increased BCR amplitude with an increase in the length of the interpulse interval might have been statistically significant if the number of patients had been larger. Moreover, the effect of the number of pulses would have been more definite under each condition. Second, we reviewed the records of patients with congenital tethered cord syndrome attributable to a few specific diagnoses (lumbosacral lipomatous malformation, limited dorsal myeloschisis, and congenital dermal sinus). We cannot guarantee that the optimal stimulation paradigm in the study will be valid for any other pathology or for healthy infants. However, if we recall that all study participants showed a clear BCR at baseline, we suppose that the results will not be very different in the case of other diagnoses if the baseline BCR is available. Third, the current study does not answer to what extent the optimal stimulation parameters contribute to improving postoperative sacral function. Although a controlled clinical trial to evaluate the utility of optimal BCR monitoring is warranted, it would be challenging to conduct given the ethical considerations and other pertinent factors, such as proficiency in surgical skills. Considering the importance 
of using neurophysiological monitoring for early intervention prior to neurological deterioration, ${ }^{10}$ we believe that optimal BCR monitoring in infants would lead to better bladder and/or bowel outcomes.

\section{Conclusions}

Biphasic 8-pulse stimulation with 5-msec intervals, followed by 2 -msec intervals, was the most effective combination among the examined conditions to generate an intraoperative BCR in infants younger than 24 months. Given the possible overlap of the BCR onset with the stimulation artifact, the interpulse interval can be shortened to a certain value between 2 and $5 \mathrm{msec}$, as needed on an individual basis. We expect these stimulation parameters to help preserve the sacral function of infants during an untethering operation.

\section{References}

1. Deletis V: Neuromonitoring, in McLone DG (ed): Pediatric Neurosurgery: Surgery of the Developing Nervous System. Philadelphia: Saunders, 2001, pp 1204-1213

2. Deletis V, Vodusek DB: Intraoperative recording of the bulbocavernosus reflex. Neurosurgery 40:88-93, 1997

3. Field-Fote EC, Anderson B, Robertson VJ, Spielholz NI: Monophasic and biphasic stimulation evoke different responses. Muscle Nerve 28:239-241, 2003

4. Hertzler DA II, DePowell JJ, Stevenson CB, Mangano FT: Tethered cord syndrome: a review of the literature from embryology to adult presentation. Neurosurg Focus 29(1):E1, 2010

5. Hoving EW, Haitsma E, Oude Ophuis CM, Journée HL: The value of intraoperative neurophysiological monitoring in tethered cord surgery. Childs Nerv Syst 27:1445-1452, 2011

6. Kothbauer KF, Novak K: Intraoperative monitoring for tethered cord surgery: an update. Neurosurg Focus 16(2):E8, 2004

7. Lee HJ, DeLisa JA: Manual of Nerve Conduction Study and Surface Anatomy for Needle Electromyography, ed 4. Philadelphia: Lippincott Williams \& Wilkins, 2004, pp 99-101
8. Lew SM, Kothbauer KF: Tethered cord syndrome: an updated review. Pediatr Neurosurg 43:236-248, 2007

9. Neuloh G, Pechstein U, Schramm J: Motor tract monitoring during insular glioma surgery. J Neurosurg 106:582-592, 2007

10. Pang D, Zovickian J, Oviedo A: Long-term outcome of total and near-total resection of spinal cord lipomas and radical reconstruction of the neural placode: part I-surgical technique. Neurosurgery 65:511-529, 2009

11. Pang D, Zovickian J, Oviedo A: Long-term outcome of total and near-total resection of spinal cord lipomas and radical reconstruction of the neural placode, part II: outcome analysis and preoperative profiling. Neurosurgery 66:253-273, 2010

12. Rinaldi F, Cioffi FA, Columbano L, Krasagakis G, Bernini FP: Tethered cord syndrome. J Neurosurg Sci 49:131-135, 2005

13. Rodi Z, Vodusek DB: Intraoperative monitoring of the bulbocavernosus reflex: the method and its problems. Clin Neurophysiol 112:879-883, 2001

14. Scheiner A, Mortimer JT, Roessmann U: Imbalanced biphasic electrical stimulation: muscle tissue damage. Ann Biomed Eng 18:407-425, 1990

\section{Disclosures}

The authors report no conflict of interest concerning the materials or methods used in this study or the findings specified in this paper.

\section{Author Contributions}

Conception and design: K Kim. Acquisition of data: Wang, SK Kim, Phi, Lee, Choi. Analysis and interpretation of data: Hwang. Drafting the article: Hwang. Critically revising the article: K Kim, Hwang. Reviewed submitted version of manuscript: all authors. Approved the final version of the manuscript on behalf of all authors: K Kim. Statistical analysis: Hwang. Study supervision: K Kim.

\section{Correspondence}

Keewon Kim, Department of Rehabilitation Medicine, Seoul National University College of Medicine, 101 Daehak-ro, Jongnogu, Seoul 110-744, Republic of Korea. e-mail: keewonkimm.d@ gmail.com. 